OPEN ACCESS

Edited by:

Jiandong Liu,

University of North Carolina at

Chapel Hill, United States

Reviewed by:

Peifeng $L i$,

Qingdao University, China

Ke Wei,

Tongji University, China

*Correspondence:

Lei Yang

lyang7@iu.edu

Specialty section:

This article was submitted to General Cardiovascular Medicine,

a section of the journal

Frontiers in Cardiovascular Medicine

Received: 22 June 2021

Accepted: 16 August 2021

Published: 16 September 2021

Citation:

Han L and Yang L (2021)

Multidimensional Mechanistic Spectrum of Long Non-coding RNAs in Heart Development and Disease. Front. Cardiovasc. Med. 8:728746

doi: $10.3389 /$ fcvm.2021.728746

\section{Multidimensional Mechanistic Spectrum of Long Non-coding RNAs in Heart Development and Disease}

\author{
Lei Han and Lei Yang* \\ Department of Pediatrics, Indiana University School of Medicine, Indianapolis, IN, United States
}

With the large-scale genome-wide sequencing, long non-coding RNAs (IncRNAs) have been found to compose of a large portion of the human transcriptome. Recent studies demonstrated the multidimensional functions of IncRNAs in heart development and disease. The subcellular localization of IncRNA is considered as a key factor that determines IncRNA function. Cytosolic IncRNAs mainly regulate mRNA stability, mRNA translation, miRNA processing and function, whereas nuclear IncRNAs epigenetically regulate chromatin remodeling, structure, and gene transcription. In this review, we summarize the molecular mechanisms of cytosolic and nuclear IncRNAs in heart development and disease separately, and emphasize the recent progress to dictate the crosstalk of cytosolic and nuclear IncRNAs in orchestrating the same biological process. Given the low evolutionary conservation of most IncRNAs, deeper understanding of human IncRNA will uncover a new layer of human regulatory mechanism underlying heart development and disease, and benefit the future clinical treatment for human heart disease.

Keywords: long non-coding RNA, microRNA, heart development, heart disease, ceRNA, PRC2 complex

\section{INTRODUCTION}

The heart is a central organ of the circulatory system, which pumps blood and drives oxygen and nutrients throughout the whole body. According to American Heart Association, heart disease is one of the leading causes of death in the United States. Approximately 655,000 Americans die of heart disease each year (1). Although heart disease could be caused by various factors, the most direct and common reason has been recognized as genetic variations in coding genes. During the past decade, accumulated evidence demonstrates that non-coding RNAs (ncRNAs) are also highly relevant to cardiovascular diseases (2). Non-coding RNAs are transcripts without prominent protein coding potential, which include two major groups, short non-coding RNAs (sncRNAs) and long non-coding RNAs (lncRNAs) (3). SncRNAs include transfer RNAs (t-RNAs), ribosomal RNAs (r-RNAs), small nuclear RNAs (snRNAs), microRNAs (miRNAs), small interfering RNAs (siRNAs) and P-element-induced wimpy testis (PIWI) interacting RNAs (piRNAs). SncRNAs are broadly involved in transcriptional and translational regulations (3). LncRNAs are over 200 bp transcripts and lncRNA genes compose a large portion of the human genome. LncRNAs display multidimensional functions at various regulatory levels, such as histone modification, DNA 
methylation, gene transcription, post-transcription, translation, RNA and protein stability (4). Many lncRNAs have been reported to be involved in cardiovascular development and disease, although their underlying molecular mechanisms in pathological process remain elusive (5). Therefore, understanding the roles of lncRNAs in heart development and disease will reveal the molecular basis of cardiogenesis, and the molecular etiology of human cardiovascular diseases. For example, a conserved lncRNA H19 represses cardiac hypertrophy by preventing and reversing experimental pressure-overload-induced heart failure, and Duchenne and Becker muscular dystrophy associated cardiomyopathy $(6,7)$. Hence, we summarize the current knowledge of characterized lncRNA mechanisms in heart development and disease (Table 1), and further discuss the clinical potential of lncRNA in heart disease therapy.

\section{LONG NON-CODING RNA FUNCTIONS IN HEART DEVELOPMENT AND DISEASE}

The establishment of in vitro cardiomyocyte (CM) differentiation from mouse and human pluripotent stem cells (hPSCs) allows modeling early events of cardiogenesis in dish. Furthermore, whole transcriptomic profiling and CRISPR/Cas-9 mediated approaches have paved the way toward discovering and functional assessment of crucial lncRNAs in early human cardiac development by using hPSCs (45). Currently, hundreds of lncRNAs have been identified in the human cardiac precursor cells (CPCs), such as cardiac mesoderm enhancerassociated non-coding RNA (Carmen), which promotes cardiac specification and differentiation of CPCs (46). A humanspecific lncRNA, Heart Brake LncRNA 1 (HBL1), represses CM differentiation from human hPSCs via counteracting $m i R$ 1 function (8). A mouse-specific lncRNA, Braveheart (Bvht), is required for the commitment of nascent mesoderm toward a cardiac fate (33). A heart field related lncRNA, Linc1405, controls cardiac mesoderm specification and cardiogenesis in mESC and in vivo (42). A lateral mesoderm-specific lncRNA Fendrr (FOXF1 Adjacent Non-Coding Developmental Regulatory RNA) plays an essential role in heart and body wall development in vivo (35). In addition to control early cardiac lineage specification, lncRNAs also play important roles in CM maturation and proliferation via various mechanisms, such as regulating the expression ratio of Myh6/Myh7 (47), sarcomere organization (43), cardiac myogenesis $(36)$, metabolic maturation $(44,48)$ and cardiac conduction $(27,31,49)$. LncRNA Mhrt (myosin heavychain-associated RNA transcripts) is required for maintaining the ratio of Myh6/Myh7 during mouse heart development and maturation, which is important for CM maturation (47). LncRNA CPR (cardiomyocyte proliferation regulator) induces

Abbreviations: lncRNA, long non-coding RNA; ceRNA, competing endogenous RNA; miRNAs microRNAs; iPSCs, induced pluripotent stem cells; CPCs, cardiac precursor cells; CM, cardiomyocyte; CAD, coronary artery disease; NICM, nonIschemic cardiomyopathy; PRC2, polycomb-repressive complex 2; AS, alternative splicing; JARID2, jumonji and AT-rich interaction domain containing 2; EED, embryonic ectoderm development; EZH2, enhancer of zeste homolog 2. hypertrophic responses of mature CMs, including increased sarcomere organization and CM surface area (43).

Evidence of the association between deregulation of lncRNAs and heart diseases has been reports for various cardiovascular disease models, such as cardiac hypotrophy (6), muscular dystrophy (7), coronary artery disease (CAD) (50-52), myocardial infarction $(32,53)$, diabetic cardiomyopathy (54), non-Ischemic cardiomyopathy (NICM) and heart failure (55). Murine and human lncRNA H19 display an anti-hypotrophy function, and CM-restricted $H 19$ gene delivery can suppress the development of cardiac hypertrophy and later on heart failure (6). Recently, Zhang et al. found that H19 inhibits dystrophin degradation, preserves skeletal and cardiac muscle histology, and improves cardiomyocyte strength and heart function in muscular dystrophy cells and murine model (7). H19 also suppresses apoptosis and autophagy of CMs under diabetic condition $(12,56)$. In myocardial infarction, lncRNA Meg3 is upregulated in infarcted mouse heart and promotes CM death (32). Although a large number of lncRNAs have been found to be associated with heart development and disease (Table 1), the mechanisms of most lncRNAs remain elusive. Particularly, the deeper understating of lncRNA mechanisms will shed light on the clinical potential of lncRNAs, with the findings of novel therapeutic targets or druggable lncRNAs. Interestingly, many lncRNAs show restricted expression patterns in the cytoplasm or nucleus although some lncRNAs express in both, suggesting the differential functions executed by lncRNAs in different subcellular localizations, which are summarized in the following sections.

\section{MECHANISMS OF LONG NON-CODING RNAS IN CYTOPLASM}

The subcellular localization is considered as a key factor determining lncRNA function $(57,58)$. Although the nucleus is the location for RNA biogenesis and processing, many mature lncRNAs are transported into cytoplasm, showing high cytosolic expressing levels (59). In the cytoplasm, lncRNAmediated mechanisms have been found to mainly regulate mRNA stability, translation of mRNA, and microRNA (miRNA) related functions (60).

\section{Long Non-coding RNA Counteracts microRNA}

Since the first discovery of competing endogenous RNA (ceRNA), hundreds of lncRNAs have been found to function as miRNA sponge to counteract endogenous miRNAs. The ceRNAs can modulate miRNA activity through sequestration, thereby increasing the expression of miRNA target genes (61). During heart development, several lncRNAs have been identified to counteract miRNAs and regulate expressions of genes essential for stem cells pluripotency or lineage specification. Using hPSCs, HBL1 was identified as a modulator to fine-tune human $\mathrm{CM}$ development via sponging miR-1 (8). HBL1 is a human-specific lncRNA highly expressed in hPSCs and gradually diminishes during CM differentiation. 
TABLE 1 | Roles of IncRNAs in heart development and diseases.

\begin{tabular}{|c|c|c|c|c|}
\hline & Cellular location & Validated target(s) & Cardiac functions & References \\
\hline \multirow[t]{2}{*}{ HBL1 } & Cytoplasm & $\operatorname{miR}-1$ & Cardiac development & $(8,9)$ \\
\hline & Nucleus & JARID2 and EED & & \\
\hline HOTAIR & Cytoplasm & $\operatorname{miR}-1$ & $\begin{array}{l}\text { Acute myocardial } \\
\text { infarction }\end{array}$ & (10) \\
\hline LINCMD1 & Cytoplasm & $\begin{array}{l}\text { miR-133; miR-135; } \\
\text { pre-miR-133b }\end{array}$ & Myogenesis & $(11)$ \\
\hline \multirow[t]{2}{*}{ H19 } & Cytoplasm & $\begin{array}{l}\text { let-7; miR-877-3p; } \\
\text { miR-22-3p; miR-19a; } \\
\text { miR-675-3p; } \\
\text { miR-675-5p; KSRP; } \\
\text { Dystrophin. }\end{array}$ & $\begin{array}{l}\text { Muscle differentiation } \\
\text { and regeneration; } \\
\text { Ml-induced myocardial } \\
\text { injury; Senescence; } \\
\text { Diabetic } \\
\text { cardiomyocyte; } \\
\text { Muscular dystrophy }\end{array}$ & $(6,7,12-20,56)$ \\
\hline & Nucleus & EED; EZH2; SUZ12 & & \\
\hline UCA1 & Cytoplasm & miR-184 & Cardiac hypertrophy & (22) \\
\hline MIAT & Cytoplasm & miR-150 & Cardiac hypertrophy & (23) \\
\hline $\mathrm{CHRF}$ & Cytoplasm & $m i R-489$ & Cardiac hypertrophy & (24) \\
\hline$R O R$ & Cytoplasm & $\operatorname{miR}-133$ & Cardiac hypertrophy & (25) \\
\hline Plscr4 & Cytoplasm & $m i R-214$ & Cardiac hypertrophy & (26) \\
\hline \multirow[t]{2}{*}{ MALAT1 } & Cytoplasm & miR-220C & $\begin{array}{l}\text { Cardiomyocyte } \\
\text { electrophysiology; } \\
\text { cardiac remodeling and } \\
\text { failure }\end{array}$ & $(27-29)$ \\
\hline & Nucleus & BRG1; HDAC9 & & \\
\hline CARL & Cytoplasm & miR-539; miR-296 & $\begin{array}{l}\text { Cardiac apoptosis, } \\
\text { replication, and } \\
\text { regeneration }\end{array}$ & (30) \\
\hline CCRR & Cytoplasm & CIP85 & Cardiac conduction & (31) \\
\hline Meg3 & Cytoplasm & FUS & Cardiac apoptosis & (32) \\
\hline Bvht & Nucleus & SUZ12 & $\begin{array}{l}\text { Cardiovascular lineage } \\
\text { commitment }\end{array}$ & $(33,34)$ \\
\hline Fendrr & Nucleus & PRC2; TrxG/MLL & $\begin{array}{l}\text { Lateral plate or cardiac } \\
\text { mesoderm } \\
\text { differentiation }\end{array}$ & (35) \\
\hline PPP1R1B & Nucleus & Ezh2 & Myogenic differentiation & (36) \\
\hline Ahit & Nucleus & SUZ12 & Cardiac hypertrophy & $(37)$ \\
\hline Chaer & Nucleus & $\mathrm{EZH} 2$ & Cardiac hypertrophy & (38) \\
\hline Uc.323 & Nucleus & $\mathrm{EZH} 2$ & Cardiac hypertrophy & (39) \\
\hline Mhrt & Nucleus & Brg1 & $\begin{array}{l}\text { Cardiac hypertrophy } \\
\text { and failure }\end{array}$ & $(41,47)$ \\
\hline Linc1405 & Nucleus & Eomes & Cardiac differentiation & $(42)$ \\
\hline CPR & Nucleus & DNMT3A & Cardiac proliferation & (43) \\
\hline \multirow[t]{2}{*}{$M D R L$} & Cytoplasm & miR-361; miR-484 & Cardiac apoptosis & (48) \\
\hline & Nucleus & Pre-miR-484 & & \\
\hline
\end{tabular}

Loss of HBL1 increases CM differentiation from hPSCs. HBL1 expresses in both nucleus and cytoplasm of undifferentiated hPSCs. In the cytoplasm, HBL1 binds with miR-1 to finetune its activity and further regulate cardiogenic gene expressions (Figure 1). Additionally, lncRNA HOTAIR (HOX antisense intergenic RNA), which was initially described as a regulator of cancer progression, also displays a cardioprotective role in acute myocardial infarction, which is partially through the interaction and negative regulation of miR-1 (10).

LINCMD1 (Long Intergenic Non-protein Coding RNA, Muscle Differentiation 1) is a muscle-specific ceRNA, which is required for muscle differentiation and plays an important role in myogenesis. LINCMD1 acts as ceRNAs for two muscle-specific microRNAs, $m i R-133$ and $m i R-135$, which target the MAML1 (expression of mastermind-like-1) and MEF2C (myocyte-specific 


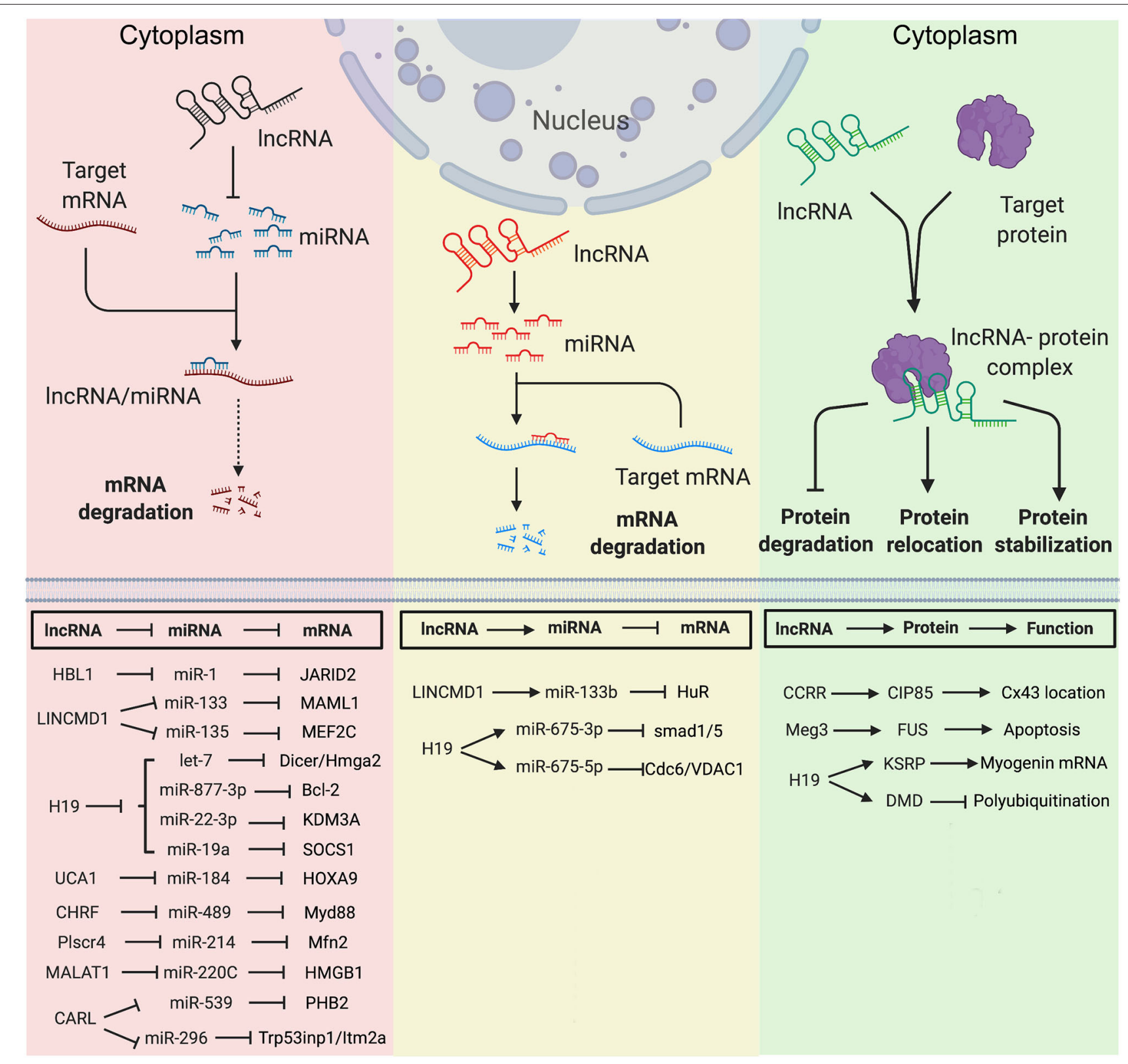

FIGURE 1 | Mechanisms of Long non-coding RNAs in cytoplasm in heart development and diseases. (Left) IncRNA functions as miRNA sponge. (Middle) IncRNA functions as miRNA precursor. (Right) IncRNA functions as protein scaffold. Created with BioRender.com.

enhancer factor 2C) genes, respectively (Figure 1). MAML1 and MEF2C are transcriptional coactivators which positively regulate muscle-specific gene expression. Depletion of LINCMD1 represses the expression of MAML1 and MEF2C, whereas overexpression of LINCMD1 increases MAML1 and MEF2C expression levels and promotes muscle differentiation (11).

It was reported that approximately 378,295 ceRNA interactions appeared in the cardiovascular disease-related ceRNA interactions (62). H19 is a lncRNA with high expression level in embryos $(63,64)$. H19 is conserved in both human and mouse and has no coding potential. H19 is required for muscle differentiation and regeneration via acting as a natural molecular sponge for the let-7 family of miRNAs (13). Depletion of H19 causes precocious muscle differentiation, which can be repressed by let-7 overexpression (Figure 1) (14). In $\mathrm{H}_{2} \mathrm{O}_{2}$-treated CMs and mouse ischemia-reperfusion (I/R) hearts, $H 19$ functions as a ceRNA for $m i R-877-3 p$, which targets Bcl-2 to further regulate mitochondria-mediated apoptosis in myocardial I/RI (Figure 1) (15). Additionally, Zhang et al. reported that H19 functions as a ceRNA of $m i R-22-3 p$, which directly targets KDM3A gene to ameliorate MI-induced myocardial injury (Figure 1) (16). H19 is also a pro-senescence lncRNA in CMs 


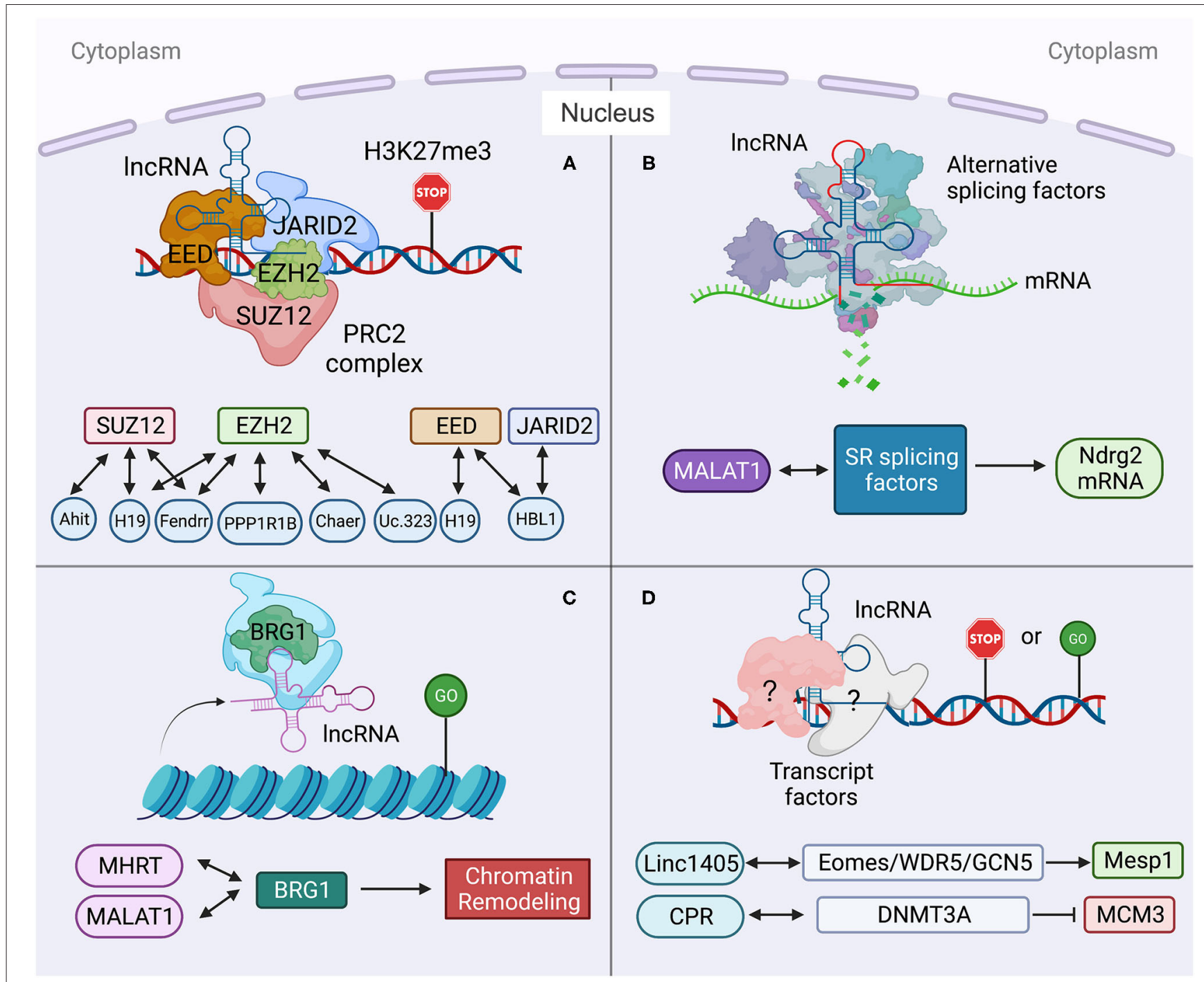

FIGURE 2 | Long non-coding RNA mechanisms in nucleus in heart development and diseases. (A) IncRNA targets to PCR2 complex and regulates its downstream gene transcription. (B) IncRNA regulates pre-mRNA alternative splicing. (C) IncRNA regulates chromatin remodeling. (D) IncRNA interacts with transcription factors to regulate gene transcription. Created with BioRender.com.

by counteracting miR-19a to upregulate SOCS1 expression and further activate the $\mathrm{p} 53 / \mathrm{p} 21$ pathway to promote CM senescence (Figure 1) (17).

Many lncRNAs have been reported to play a ceRNA role in hypertrophic cardiomyopathy. LncRNA UCA1 regulates cardiac hypertrophy via the UCA1/miR-184/HOXA9 axis (Figure 1) (22). MIAT promotes cardiac hypertrophy through targeting miR-150 (23). LncRNA CHRF (cardiac hypertrophy related factor) regulates cardiac hypertrophy via the $C H R F / m i R$ 489/Myd88 axis (24). LncRNA ROR mediates cardiac remodeling and promotes cardiac hypertrophy via interacting with $m i R-133$ (25). Plscr4 negatively regulates cardiac hypertrophy in vivo and in vitro via the $m i R-214 / \mathrm{Mfn} 2$ axis (26). MALAT1 (metastasisassociated lung adenocarcinoma transcript 1) reduces transient outward potassium current of CMs by targeting $m i R-220 \mathrm{C}$ and its downstream target gene HMGB1 (Figure 1) (27). CARL (cardiac apoptosis-related lncRNA) significantly increases in CMs since the neonatal stage of mouse (44). CARL can negatively regulate mitochondrial fission and apoptosis through the $m i R-539 /$ PHB2 axis (44). It can also directly target $m i R$ 296 and its downstream genes Trp53inp1 and Itm2a, further regulating $\mathrm{CM}$ replication and cardiac regeneration after injury (Figure 1) (30).

To date, hundreds of publications have reported the ceRNA role of lncRNAs under normal and diseased conditions. Given the cascading effects exerted by the gene networks comprising ceRNA-miRNAcoding genes, lncRNA and its downstream gene networks are potential new targets for cardiovascular disease therapy. 


\section{Long Non-coding RNA Forms miRNA Precursor}

LncRNAs can be transcribed as miRNA precursors, which produce mature miRNAs via further processing. Therefore, lncRNAs could indirectly regulate the expression of miRNA downstream target genes. For example, transcript of LINCMD1 hosts a pre-miR-133b transcript. The RNA-binding protein HuR is a component of LINCMD regulatory circuitry to regulate muscle differentiation (65). During the early stage of muscle differentiation, HuR binds to LINCMD1 and promotes miR133 biogenesis from the LINCMD1 transcript. HuR/LINCMD1 complex is then targeted by $m i R-133$ in the cytoplasm (Figure 1). Thus, the ceRNA function of LINCMD1 reinforces HuR expression via counteracting $m i R-133$ in a positive feedforward loop (65). In this case, LINCMD1 plays dual roles in fine-tuning the dynamic of muscle differentiation and regeneration.

Interestingly, the exon 1 of $H 19$ hosts transcripts of miR-675-3p and miR-675-5p. MiR-675-3p regulates the bone morphogenetic protein (BMP) signaling pathway by directly targeting Smad1 and Smad5 mRNAs (Figure 1) (18). MiR-675$5 p$ could target DNA replication initiation factor Cdc6 mRNA (18). Therefore, H19 exhibits a pro-differentiation function in primary myoblasts and regenerating skeletal muscles (19). In the rat model of diabetic cardiomyopathy, overexpression of $H 19$ can attenuate apoptosis of diabetic CMs and improve left ventricular function, whereas knockdown of $\mathrm{H} 19$ shows opposite functions. Mechanistically, H19 expression is significant downregulated in the hearts of rats with diabetic cardiomyopathy, which leads to a reduced level of $m i R-675$ and an increased level of $m i R-675$ target-gene VDAC1. Enhanced VDAC1 can induce apoptosis of CMs when exposed to high glucose (12).

\section{Long Non-coding RNA Functions as Protein Scaffold}

In cytoplasm, IncRNA can regulate protein location and stability by directly binding with target protein(s). As an anti-arrhythmic lncRNA, CCRR (cardiac conduction regulatory RNA) is downregulated in both mouse and human heart failure (31). CCRR knockdown induces arrhythmias, and its overexpression improves cardiac conduction. CCRR is also required for maintaining the proper distribution of connexin 43 (CX43) in the intercalated discs (Figure 1). Mechanically, CCRR directly binds with CX43-interacting protein CIP85 and prevents CX43 from backward trafficking and subsequent degradation in the cytoplasm of CMs (31).

$M e g 3$ is upregulated in infarcted mouse hearts and human failing hearts. Meg3 expression is directly regulated by p53 under hypoxic condition. It has been reported that $\mathrm{Meg} 3 \mathrm{has}$ a proapoptotic function in rodent CMs (32). Meg3 shRNA delivered by the adeno-associated virus serotype 9 (AAV9) can significantly improve cardiac function. Meg3 functions as protein scaffold to direct bind with RNA-binding protein FUS and regulates apoptotic signaling pathway (Figure 1) (32).

Except for the functions mentioned above, H19 also interacts with proteins in the cytoplasm. In the undifferentiated multipotent mesenchymal $\mathrm{C} 2 \mathrm{C} 12$ cells, H19 interacts with a multifunctional RNA binding protein KSRP (K homologytype splicing regulatory protein) (20). To maintain the undifferentiated state of $\mathrm{C} 2 \mathrm{C} 12$ cells, cytoplasmic $H 19$ posttranscriptionally modulates gene expression via acting as a protein scaffold of KSRP and promotes its interaction with RNA exosome, which further enhances the KSRP-promoted mRNA decay of myogenic genes (20). Recently, in muscular dystrophy (MD) patients, H19 was found to directly interact with dystrophin and inhibit E3-ligase-dependent polyubiquitination at Lys3584 for protein degradation. Non-silent mutation (C3340Y) of dystrophin results in defective interaction between dystrophin and H19, which causes ubiquitination and degradation of dystrophin (Figure 1) (7). In both Dmd mouse model and human iPSC-derived skeletal muscle cells from patients with Becker MD, simultaneous administration of H19 RNA mimic and nifenazone, an analgesic for rheumatic conditions, could effectively inhibit dystrophin degradation, preserve skeletal and cardiac muscle histology, and improve cardiac strength and heart function. This suggests a protective role of H19 in both Becker and Duchenne muscular dystrophy, providing a potential RNA therapy for MD patients (7).

\section{LONG NON-CODING RNA FUNCTIONS IN NUCLEUS}

Compared to cytoplasm, RNAs are processed in nucleus where many lncRNAs reside and execute functions. Nuclear lncRNAs play a variety of crucial roles with complex molecular mechanisms, including regulating chromatin organization, transcription, and different nuclear condensates (66).

\section{Long Non-coding RNA Interacts With the Polycomb-Repressive Complex 2 (PRC2)}

Multiple nuclear lncRNAs have been found to regulate lineage differentiation by interacting with PRC2. Histone-modifying complex PRC2 plays a pivotal role in determining the epigenetic state of genes controlling pluripotency, lineage commitment, and cell differentiation (67). A heart-associated lncRNA, Bvht is required for the commitment of nascent mesoderm to a cardiac fate from mouse ESCs (33). In the nucleus, Bvht can activate the core cardiovascular gene network by interacting with SUZ12, a component of PRC2, during CM differentiation (Figure 2). In Bvht-depleted cells, SUZ12 and PRC2 associated chromatin modification $\mathrm{H} 3 \mathrm{~K} 27 \mathrm{me} 3$ are deposited at promoters of cardiogenic genes, such as Mesp1, which is a master regulator of cardiovascular fate commitment (33). Additionally, deletion of a $5^{\prime}$ asymmetric G-rich internal loop (AGIL) in Bvht can dramatically impair CM differentiation (34). Through AGIL, $B v h t$ can interact with a cellular nucleic acid binding protein CNBP (ZNF9), which is known as a zinc-finger protein to bind with single-stranded G-rich sequences. Together, in the nucleus, $B v h t$ controls cardiovascular lineage commitment by interacting with SUZ12/PRC2 and CNBP through defined RNA motifs (33, 34).

In mouse, a lateral mesoderm-specific lncRNA Fendrr is essential for heart development (35). During mouse embryo 
development, Fendrr binds with both PRC2 via the EZH2 subunit and TrxG/MLL complexes and acts as modulators of PRC2 or TrxG/MLL activity (Figure 2) (35). Fendrr deficient embryos show upregulation of several transcription factors controlling lateral plate or cardiac mesoderm differentiation, accompanied with a drastic reduction of PRC2 occupancy and decreased $\mathrm{H} 3 \mathrm{~K} 27$ trimethylation and/or increased $\mathrm{H} 3 \mathrm{~K} 4$ trimethylation at those gene promoters. So, similar to Bvht, Fendrr plays an essential role in controlling cardiac lineage fate commitment via PRC2 (35).

The interaction between lncRNA and PRC2 complex is conserved in human and mouse. LncRNA PPP1R1B was found to bind with EZH2, a key PRC2 subunit (Figure 2) (36, 68). Silencing of PPP1R1B compromises myotube development in both mouse $\mathrm{C} 2 \mathrm{C} 12$ and human skeletal myoblasts (36). In hiPSCs-CMs, $P P P 1 R 1 B$ deficient also impairs myogenic differentiation (36). $P P P 1 R 1 B$ regulates the expression of myogenic transcription factors, such as MyoD, Myogenin, and Tbx5, by interacting with PRC2 at the chromatin interface. PPP1R1B interacts with PRC2 to suppress H3K27me3 histone modification on the MyoD1 and Myogenin promoters. In the nucleus, $P P P 1 R 1 B$ modulates PRC2 occupancy on promoters of essential myogenic genes to regulate myogenic differentiation during heart and skeletal muscle development (36).

Our recent study found that nuclear $H B L 1$ interacts with two PRC2 subunits, JARID2 and EED in human pluripotent stem cells (Figure 2) (9). During human cardiogenesis, loss of HBL1 disrupts genome-wide PRC2 occupancy, reduces H3K27me3 chromatin modification on essential cardiogenic genes, and therefore enhances cardiogenic gene transcription in undifferentiated hPSCs and later-on differentiation. At the pluripotency stage, deletions of HBL1 and JARID2 both reduce PRC2 occupancy on 62 overlapped cardiogenic genes. Therefore, $H B L 1$ precisely controls cardiogenic gene transcription via modulating PRC2 occupancy.

H19 plays important functions in both cytoplasm and nucleus. In diabetic cardiomyopathy, cytosolic H19 forms miR-675-3p and $m i R-675-5 p$ and attenuates apoptosis of CMs (12). Under the same pathological condition, Zhuo et al. reported that H19 directly binds with EZH2, a subunit of PRC2, in CM nucleus to affect the anti-autophagy function (56). Loss of H19 was found to reduce EZH2 and H3K27me3 occupancy on the promoter of DIRAS3, which regulates the formation of autophagosome initiation complex (Figure 2) (21), and causes DIRAS3 downregulation. Consistent with its cytosolic function (12), overexpression of H19 can inhibit cell death of CMs caused by high glucose via this nuclear mechanism. Recently, Viereck et al. reported the interaction between H19 and PRC2 complex subunits EED, EZH2 and SUZ12 in the nuclear lysate of HL-1 CMs (Figure 2) (6). In pressure overload-induced left ventricular hypertrophy mice, $\mathrm{H} 19$ ablation aggravates cardiac hypertrophy compared to wild-type mice. Taken together, H19 physically interacts with PRC2 to suppress $\mathrm{H} 3 \mathrm{~K} 27 \mathrm{me} 3$ modification at the Tescalcin locus, which is an anti-hypertrophic gene, to promote Tescalcin expression and in turn repress the NFAT signaling pathway (6).
Many other lncRNAs also have been found to interact with PRC2 complex under heart disease conditions. Ahit suppresses cardiac hypertrophy through binding with SUZ12 to regulate PRC2 occupancy on the MEF2A (myocyte enhancer factor 2A) promoter (Figure 2) (37). Chaer is required for the development of cardiac hypertrophy through direct binding with PRC2 subunit EZH2 to further regulate expressions of Anf, Myh7 and Actal genes (Figure 2) (38). Uc.323 protects CMs against cardiac hypertrophy by binding with EZH2 to regulate $\mathrm{CPT} 1 \mathrm{~b}$ gene expression (Figure 2) (39).

Taken together, IncRNAs play important roles in cardiac development and diseases by interacting with PRC2 complex to affect PRC2-related epigenetic modifications.

\section{Long Non-coding RNA Regulates Alternative Splicing of Pre-mRNA}

Alternative splicing (AS) of pre-mRNA enhances diversities of transcriptome and proteomic of the genome in higher eukaryotes (69). During tissue- or cell-type specification, the serine/arginine (SR) splicing factors regulate AS in a concentration or phosphorylation dependent manner $(70,71)$. During human cardiovascular differentiation, stage-specific RNA alternative splicing and lineage-enriched lncRNAs were identified by whole RNA-seq (45). As a long nuclear-retained regulatory RNA (nrRNA), MALAT1 interacts with SR splicing factors in the nuclear speckle domains (Figure 2) (28). MALAT1 regulates mRNA alternative splicing by modulating the levels of phosphorylated SR proteins (28). During pressure overloadinduced cardiac remodeling and failure, Malat1 was found to be an alternative splicing regulator of $\mathrm{Ndrg} 2$, which shows skipped exon 3 in hypertrophic mouse hearts $(72,73)$.

\section{Long Non-coding RNA and Chromatin Remodeling}

In addition to interactions with splicing factors and epigenetic factors, lncRNAs have also been shown to interact with chromatin remodeling complexes (74). Mhrt (myosin heavychain-associated RNA transcripts) is a cardiac-specific lncRNA located in the murine myosin heavy chain 7 locus and is suppressed by the BRG1-HDAC-PARP chromatin repressor complex in cardiomyopathy (75). Overexpression of Mhrt protects mouse heart from hypertrophy and failure (47). Mhrt directly binds with BRG1, which is a chromatin-remodeling factor and the ATPase subunit of the SWI/SNF complex (Figure 2) (40), to remove SWI/SNF from its occupied genomic regions on target genes, thus regulating chromatin remodeling and gene transcription. Mhrt binds with the helicase domain of BRG1, which is crucial for tethering BRG1to its targets. In turn, BRG1 represses Mhrt in stress-induced cardiac hypertrophy and failure (41). This MHRT-BRG1 feedback circuit is also conserved in the human heart (47). MALAT1 can also form RNA-protein complex with chromatin-remodeling enzyme BRG1 and histone deacetylase HDAC9 in vascular smooth muscle cells (Figure 2). This HDAC9-MALAT1-BRG1 complex represses expression of contractile protein genes in association with gain of $\mathrm{H} 3 \mathrm{~K} 27 \mathrm{me} 3$ histone modification (29). 


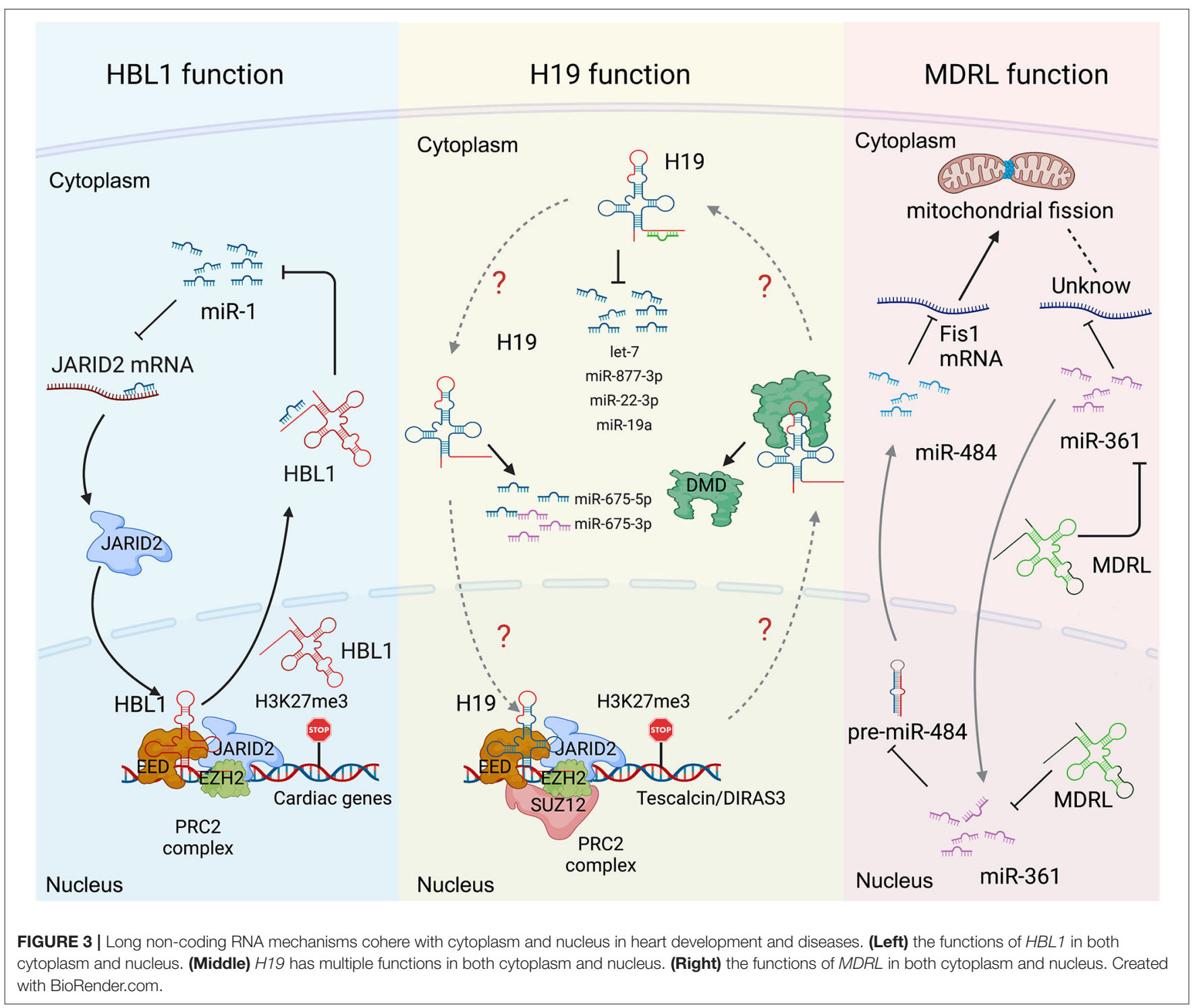

\section{Long Non-coding RNA Interacts With Transcription Factors}

Besides chromatin-remodeling factors and epigenetic factors, transcription factors have also been found to interact with lncRNAs in heart development and disease. Linc1405 is highly expressed in heart during mouse embryo development and critical for proper cardiac differentiation (42). Linc1405 interdependently interacts with Eomes, which physically mediates Eomes/WDR5/GCN5 complex binding at the enhancer region of Mesp1 gene to activate its expression (Figure 2) (42). Mesp1 is one of the earliest key regulators of cardiac lineage specification (76). Disruption of Mesp1 in mice results in embryonic lethality due to a cardiac mesoderm deficiency (77). Therefore, linc1405 guides Eomes/WDR5/GCN5 complex to directly target Mesp1 and affect expression of Mesp1 downstream genes to control cardiac differentiation (42).
Recently, lncRNA CPR (cardiomyocyte proliferation regulator) was found to play an important role in the regulation of CM proliferation (43). Deletion of CPR in CMs increases $\mathrm{CM}$ proliferation, reduces scar formation, and improves heart function after myocardial injury. Mechanically, $C P R$ represses CM proliferation by suppressing the transcription of MCM3, which regulates initiation of eukaryotic genome replication and cell cycle (78) by direct binding with DNMT3A. Further, DNMT3A promotes CpG methylation of MCM3 promoter and represses transcription of MCM3 (Figure 2) (43).

\section{CROSSTALK OF CYTOSOLIC AND NUCLEAR PORTIONS OF THE SAME LONG NON-CODING RNA}

Many lncRNAs, such as $\operatorname{HBL1}(8,9), H 19(6,7,13,14,19,20)$, $M D R L$ (48) and LncMyoD (79), express in both cytoplasm and 
nucleus to display different functional mechanisms. However, how the cytosolic and nuclear mechanisms mediated by the same lncRNA could crosstalk with each other has been rarely studied. Recently, we reported the function of nuclear HBL1 in human cardiogenesis (9), following our previous characterization of cytosolic HBL1 role during human CM differentiating (8). We also defined the mechanism by which cytosolic and nuclear $H B L 1$ crosstalk to control cardiogenic gene transcription (9). $H B L 1$ functions as a $m i R-1$ sponge in cytoplasm and governs $P R C 2$ occupancy on cardiogenic genes in nucleus (Figure 3). In the meanwhile, miR-1 was found to bind with 3'UTR of JARID2 mRNA to repress its expression, and JARID2 deficiency reduces PRC2 occupancy on cardiogenic genes. This conserved miR-1-JARID2 axis thus allows precise regulation of nuclear PRC2 occupancy on cardiogenic genes through miR-1 activity in cytosol (Figure 3). In the cytoplasm, HBL1 counteracts $m i R-1$, which further determines mRNA and protein level of JARID2. After JARID2 protein entering nucleus, nuclear HBL1 binds with JARID2 and EED to determine PRC2 occupancy on cardiogenic genes (Figure 3). Together, this $H B L 1 / m i R$ 1-HBL1/JARID2/PRC2 mechanism coordinates to fine-tune the chromatin state of essential cardiogenic genes in human cardiogenesis $(8,9)$.

H19 has been well-studied in heart development and various heart diseases, including diabetic cardiomyopathy (12, 56), myocardial infarction (15), cardiac hypertrophy (6), muscular dystrophies (7) and heart failure (17). H19 displays multiple functions in both cytoplasm and nucleus. Under cardiac hypertrophy, $H 19$ acts as a $m i R-675$ precursor to regulate the expression of miR-675 downstream gene VDAC1 and CM apoptosis in cytoplasm (Figure 3) (12); In nucleus, H19 regulates PRC2 occupancy on the promoters of DIRAS3 and Tescalcin to repress cardiac hypotrophy (Figure 3) $(6,56)$. Consistently, all those studies reported that overexpression of H19 in CMs can mitigate cardiac hypertrophy $(6,12,56)$. These observations raise a question whether the cytosolic and nuclear functions of H19 could coordinate to regulate cardiac hypertrophy, which remains to be further investigated.

MDRL (mitochondrial dynamic related lncRNA) is another well-studied lncRNA with defined mechanisms in both nucleus and cytoplasm. MDRL functions as a ceRNA of $m i R-361$, which directly affects $m i R-484$ expression in mouse CMs (Figure 3) (48). $M D R L$ inhibits mitochondrial fission and apoptosis through two miRNAs, $m i R-361$ and $m i R-484$. In nucleus, $M D R L$ affects the processing of pre-miR-484 by targeting miR-361. In cytoplasm, $M D R L$ regulates the mitochondrial network through both $m i R-361$ and $m i R-484$ (Figure 3). This work defined the complex functions of MDRL in both miRNA processing and downstream gene expression (48). All these findings suggest that clinical application of lncRNA should rely on deeper mechanistic studies, especially the differential roles of the same lncRNA in nucleus and cytoplasm.

\section{CONCLUSIONS}

We summarized the biological functions and molecular mechanisms of lncRNAs in heart development and disease.
In heart development, lncRNAs Carmen, HBL1, Bvht, Fendrr, Bvht and CRP regulate cardiac fate commitment, lineage differentiation, CM maturation/proliferation, and sarcomere organization etc. via both nuclear and cytoplasmic mechanisms. In heart diseases, lncRNAs are involved in the pathogenesis of cardiac hypotrophy, muscular dystrophy, myocardial infarction, diabetic cardiomyopathy, nonIschemic cardiomyopathy (NICM) and heart failure and so on. With current progresses of genome-wide sequencing and functional screening studies, more functional lncRNAs have been identified in organogenesis and diseases, although the detailed molecular mechanisms of most lncRNAs have not been clearly defined. For example, IncRNAs ALIEN is expressed in undifferentiated pluripotent stem cells and impairs cardiovascular differentiation from pluripotent stem cells with molecular mechanism to be further studied (80). LncRNA GASL1 is downregulated in chronic heart failure and can inhibit CM apoptosis through TGF- $\beta 1$ signaling pathway, but how it regulates TGF- $\beta 1$ is unclear (81). A group of lncRNAs are enriched in peripheral blood under different heart disease conditions (82, 83). For example, lncRNA Heat2 expression is increased in the blood of heart failure patients (84); lncRNA MT-LIPCAR, transcribed from mitochondrial DNA, is positively associated with left ventricular diastolic dysfunction $(54,85)$. Although these lncRNAs might be utilized as disease markers or possess therapeutic penitential, their molecular mechanisms still require further characterizations.

The subcellular location of lncRNA is critical for its function, particularly for those lncRNAs highly expressed in both nucleus and cytoplasm (86). Cytosolic lncRNAs mainly function as regulators of mRNA stability, mRNA translation, miRNA processing and function, whereas nuclear lncRNAs can epigenetically regulate chromatin remodeling, structure, and gene transcription. Therefore, the balanced doses and transportation of lncRNA between cytoplasm and nucleus are expected to be a new research topic in the lncRNA field. During the last two decades, the translational potential of noncoding RNAs in heart disease therapy has gradually emerged. Nowadays, accumulated evidence indicates that $\ln \mathrm{R}$ RAs provide a new layer of regulatory mechanism on top of coding genes. Since many lncRNAs have low evolutionary conservation (87), studies of lncRNAs might also reveal unique molecular mechanisms of heart development and disease in the human. Given the complex mechanisms, it is expected lncRNAs could offer new preventive and treatment approaches for human diseases including cardiovascular disease. Although, currently, there is no lncRNA therapeutic approach has progressed into preclinical or clinical trial, H19 has been tested as a potential clinical therapeutic target in the Yucatan mini-pig (88). The expression changes of lncRNAs under different setting of heart diseases make it difficult for clinical applications. For example, in cardiac hypertrophy, Mhrt is downregulated (47), while Chaer and Chrf are upregulated (24, 38). MALAT1 and Whispr expressions are upregulated in cardiac fibrosis, whereas Meg3 and GAS5 expressions are downregulated (89-92). Nevertheless, upregulated lncRNAs can 
be repressed by using shRNA, locked nucleic acids (LNAs) or GapmeR, and downregulated lncRNAs can be enhanced by using virus such as adenovirus, adeno-associated virus (AAV), and lentivirus (93). Although no clinical trial exists for lncRNA therapy in heart disease, the success of non-coding RNA miR-132 based clinical trial paved the way. Recently, phase $1 \mathrm{~b}$ clinical study to assess safety, pharmacokinetics and pharmacodynamics parameters of CDR132L, a miR-132 inhibitor, has been completed (94). CDR132L is safe and well tolerated. Importantly, it improves cardiac function of heart failure patients. Therefore, the clinical applications of lncRNAs have a bright future, with fully and clearly characterized molecular mechanisms.

\section{REFERENCES}

1. Virani SS, Alonso A, Aparicio HJ, Benjamin EJ, Bittencourt MS, Callaway CW, et al. Heart disease and stroke statistics-2021 update: a report from the american heart association. Circulation. (2021) 143:e254-e743. doi: 10.1161/CIR.0000000000000950

2. Das S, Shah R, Dimmeler S, Freedman JE, Holley C, Lee JM, et al. Noncoding RNAs in cardiovascular disease: current knowledge, tools and technologies for investigation, and future directions: a scientific statement from the american heart association. Circ Genom Precis Med. (2020) 13:e000062. doi: 10.1161/HCG.0000000000000062

3. Zhang $\mathrm{P}, \mathrm{Wu} \mathrm{W}$, Chen $\mathrm{Q}$, Chen $\mathrm{M}$. Non-coding RNAs and their integrated networks. J Integr Bioinform. (2019) 16:20190027. doi: 10.1515/jib-2019-0027

4. Statello L, Guo CJ, Chen LL, Huarte M. Gene regulation by long noncoding RNAs and its biological functions. Nat Rev Mol Cell Biol. (2021) 22:96-118. doi: 10.1038/s41580-020-00315-9

5. Gomes CPC, Spencer H, Ford KL, Michel LYM, Baker AH, Emanueli C, et al. The function and therapeutic potential of long non-coding RNAs in cardiovascular development and disease. Mol Ther Nucleic Acids. (2017) 8:494-507. doi: 10.1016/j.omtn.2017.07.014

6. Viereck J, Buhrke A, Foinquinos A, Chatterjee S, Kleeberger JA, Xiao K, et al. Targeting muscle-enriched long non-coding RNA H19 reverses pathological cardiac hypertrophy. Eur Heart J. (2020) 41:3462-74. doi: 10.1093/eurheartj/ehaa519

7. Zhang Y, Li Y, Hu Q, Xi Y, Xing Z, Zhang Z, et al. The lncRNA H19 alleviates muscular dystrophy by stabilizing dystrophin. Nat Cell Biol. (2020) 22:1332-45. doi: 10.1038/s41556-020-00595-5

8. Liu J, Li Y, Lin B, Sheng Y, Yang L. HBL1 is a human long noncoding RNA that modulates cardiomyocyte development from pluripotent stem cells by counteracting MIR1. Dev Cell. (2017) 42:333-348 e5. doi: 10.1016/j.devcel.2017.07.023

9. Liu J, Liu S, Han L, Sheng Y, Zhang Y, Kim IM, et al. LncRNA HBL1 is required for genome-wide PRC2 occupancy and function in cardiogenesis from human pluripotent stem cells. Development. (2021) 148:dev199628. doi: 10.1242/dev.199628

10. Gao L, Liu Y, Guo S, Yao R, Wu L, Xiao L, et al. Circulating long noncoding RNA HOTAIR is an essential mediator of acute myocardial infarction. Cell Physiol Biochem. (2017) 44:1497-508. doi: 10.1159/000485588

11. Cesana M, Cacchiarelli D, Legnini I, Santini T, Sthandier O, Chinappi M. et al. A long noncoding RNA controls muscle differentiation by functioning as a competing endogenous. RNCell A. (2011) 147:35869. doi: 10.1016/j.cell.2011.09.028

12. Li $\mathrm{X}$, Wang $\mathrm{H}$, Yao $\mathrm{B}$, Xu W, Chen J, Zhou $\mathrm{X}$. IncRNA H19/miR-675 axis regulates cardiomyocyte apoptosis by targeting VDAC1 in diabetic cardiomyopathy. Sci Rep. (2016) 6:3 6340. doi: 10.1038/srep36340

13. Poirier F, Chan C, Timmons P, Robertson E, Evans M, Rigby P. The murine $\mathrm{H} 19$ gene is activated during embryonic stem cell differentiation in vitro and

\section{AUTHOR CONTRIBUTIONS}

LH and LY summarized the references and prepared the manuscript. LH drafted the illustrations. LY supervised the project. All authors contributed to the article and approved the submitted version.

\section{FUNDING}

This was supported by NIH (RO1 HL 147871, R21 HD095049) and AHA (20EIA35260114, 19TPA34850038) to LY, and 2019 AHA postdoc fellowship Award (19POST34380871) to LH.

at the time of implantation in the developing embryo. Development. (1991) 113:1105-14. doi: 10.1242/dev.113.4.1105

14. Kallen AN, Zhou XB, Xu J, Qiao C, Ma J, Yan L, et al. The imprinted H19 lncRNA antagonizes let-7 microRNAs. Mol Cell. (2013) 52:10112. doi: 10.1016/j.molcel.2013.08.027

15. Li X, Luo S, Zhang J, Yuan Y, Jiang $W$, Zhu $H$, et al. IncRNA H19 alleviated myocardial I/RI via suppressing miR-877-3p/Bcl-2mediated mitochondrial apoptosis. Mol Ther Nucleic Acids. (2019) 17:297-309. doi: 10.1016/j.omtn.2019.05.031

16. Zhang BF, Jiang $\mathrm{H}$, Chen J, Hu Q, Yang S, Liu XP, et al. LncRNA H19 ameliorates myocardial infarction-induced myocardial injury and maladaptive cardiac remodelling by regulating KDM3A. J Cell Mol Med. (2020) 24:1099-115. doi: 10.1111/jcmm.14846

17. Zhuang Y, Li T, Xiao H, Wu J, Su S, Dong X, et al. LncRNA-H19 drives cardiomyocyte senescence by targeting miR-19a/socs $1 / \mathrm{p} 53$ axis. Front Pharmacol. (2021) 12:631835. doi: 10.3389/fphar.2021.63 1835

18. Dey BK, Pfeifer K, Dutta A. The H19 long noncoding RNA gives rise to microRNAs miR-675-3p and miR-675-5p to promote skeletal muscle differentiation and regeneration. Genes Dev. (2014) 28:491501. doi: 10.1101/gad.234419.113

19. Cai X, Cullen BR. The imprinted H19 noncoding RNA is a primary microRNA precursor. RNA. (2007) 13:313-6. doi: 10.1261/rna.351707

20. Giovarelli M, Bucci G, Ramos A, Bordo D, Wilusz CJ, Chen CY, et al. H19 long noncoding RNA controls the mRNA decay promoting function of KSRP. Proc Natl Acad Sci U S A. (2014) 111:E5023-8. doi: 10.1073/pnas.1415098111

21. Lu Z, Baquero MT, Yang H, Yang M, Reger AS, Kim C, et al. DIRAS3 regulates the autophagosome initiation complex in dormant ovarian cancer cells. Autophagy. (2014) 10:1071-92. doi: 10.4161/auto.28577

22. Zhou G, Li C, Feng J, Zhang J, Fang Y. lncRNA UCA1 Is a Novel regulator in cardiomyocyte hypertrophy through targeting the miR184/HOXA9 axis. Cardiorenal Med. (2018) 8:130-9. doi: 10.1159/0004 87204

23. Zhu X, Yuan Y, Rao S, Wang P. LncRNA MIAT enhances cardiac hypertrophy partly through sponging miR-150. Eur Rev Med Pharmacol Sci. (2016) 20:3653.

24. Wang K, Liu F, Zhou LY, Long B, Yuan SM, Wang Y, et al. The long noncoding RNA CHRF regulates cardiac hypertrophy by targeting miR-489. Circ Res. (2014) 114:1377-88. doi: 10.1161/CIRCRESAHA.114.3 02476

25. Jiang F, Zhou X, Huang J. Long non-coding RNA-ROR mediates the reprogramming in cardiac hypertrophy. PLoS ONE. (2016) 11:e0152767. doi: 10.1371/journal.pone.0152767

26. Lv L, Li T, Li X, Xu C, Liu Q, Jiang H, et al. The lncRNA Plscr4 controls cardiac hypertrophy by regulating miR-214. Mol Ther Nucleic Acids. (2018) 10:387-97. doi: 10.1016/j.omtn.2017.12.018

27. Zhu P, Yang M, Ren H, Shen G, Chen J, Zhang J, et al. Long noncoding RNA MALAT1 downregulates cardiac transient outward potassium current 
by regulating miR-200c/HMGB1 pathway. J Cell Biochem. (2018) 119:1023949. doi: $10.1002 /$ jcb. 27366

28. Tripathi V, Ellis JD, Shen Z, Song DY, Pan Q, Watt AT, et al. The nuclear-retained noncoding RNA MALAT1 regulates alternative splicing by modulating SR splicing factor phosphorylation. Mol Cell. (2010) 39:92538. doi: 10.1016/j.molcel.2010.08.011

29. C.L. Lino Cardenas, Kessinger CW, Cheng Y, MacDonald C, MacGillivray T, Ghoshhajra B, et al. An HDAC9-MALAT1-BRG1 complex mediates smooth muscle dysfunction in thoracic aortic aneurysm. Nat Commun. (2018) 9:1009. doi: 10.1038/s41467-018-03394-7

30. Cai B, Ma W, Ding F, Zhang L, Huang Q, Wang X, et al. The long noncoding RNA CAREL controls cardiac regeneration. J Am Coll Cardiol. (2018) 72:53450. doi: 10.1016/j.jacc.2018.04.085

31. Zhang Y, Sun L, Xuan L, Pan Z, Hu X, Liu H, et al. Long non-coding RNA CCRR controls cardiac conduction via regulating intercellular coupling. Nat Commun. (2018) 9:4176. doi: 10.1038/s41467-018-06637-9

32. Wu H, Zhao ZA, Liu J, Hao K, Yu Y, Han X, et al. Long noncoding RNA Meg3 regulates cardiomyocyte apoptosis in myocardial infarction. Gene Ther. (2018) 25:511-23. doi: 10.1038/s41434-018-0045-4

33. Klattenhoff CA, Scheuermann JC, Surface LE, Bradley RK, Fields $\mathrm{PA}$, Steinhauser ML, et al. Braveheart, a long noncoding RNA required for cardiovascular lineage commitment. Cell. (2013) 152:570-83. doi: 10.1016/j.cell.2013.01.003

34. Xue Z, Hennelly S, Doyle B, Gulati AA, Novikova IV, Sanbonmatsu KY, et al. A G-rich motif in the IncRNA braveheart interacts with a zinc-finger transcription factor to specify the cardiovascular lineage. Mol Cell. (2016) 64:37-50. doi: 10.1016/j.molcel.2016.08.010

35. Grote P, Wittler L, Hendrix D, Koch F, Wahrisch S, Beisaw A, et al. The tissue-specific lncRNA Fendrr is an essential regulator of heart and body wall development in the mouse. Dev Cell. (2013) 24:20614. doi: 10.1016/j.devcel.2012.12.012

36. Kang X, Zhao Y, Van Arsdell G, Nelson SF, Touma M. Ppplr1b-lncRNA inhibits PRC2 at myogenic regulatory genes to promote cardiac and skeletal muscle development in mouse and human. RNA. (2020) 26:48191. doi: 10.1261/rna.073692.119

37. Yu J, Yang $\mathrm{Y}, \mathrm{Xu} \mathrm{Z}$, Lan $\mathrm{C}$, Chen $\mathrm{C}$, Li C, et al. Long noncoding RNA ahit protects against cardiac hypertrophy through SUZ12 (Suppressor of Zeste 12 Protein Homolog)-mediated downregulation of MEF2A (Myocyte Enhancer Factor 2A). Circ Heart Fail. (2020) 13:e006525. doi: 10.1161/CIRCHEARTFAILURE.119.006525

38. Wang Z, Zhang XJ Ji YX, Zhang P, Deng KQ, Gong J, et al. The long noncoding RNA Chaer defines an epigenetic checkpoint in cardiac hypertrophy. Nat Med. (2016) 22:1131-9. doi: 10.1038/nm.4179

39. Sun Y, Fan $W$, Xue R, Dong B, Liang $Z$, Chen $C$, et al. Transcribed ultraconserved regions, Uc323, ameliorates cardiac hypertrophy by regulating the transcription of CPT1b (Carnitine Palmitoyl transferase 1b). Hypertension. (2020) 75:79-90. doi: 10.1161/HYPERTENSIONAHA.119.13173

40. Alver BH, Kim KH, Lu P, Wang X, Manchester HE, Wang W, et al. The SWI/SNF chromatin remodelling complex is required for maintenance of lineage specific enhancers. Nat Commun. (2017) 8:14648. doi: $10.1038 /$ ncomms 14648

41. Han P, Chang CP. Long non-coding RNA and chromatin remodeling. RNA Biol. (2015) 12:1094-8. doi: 10.1080/15476286.2015.1063770

42. Guo X, Xu Y, Wang Z, Wu Y, Chen J, Wang G, et al. A Linc1405/Eomes complex promotes cardiac mesoderm specification and cardiogenesis. Cell Stem Cell. (2018) 22:893-908 e6. doi: 10.1016/j.stem.2018.04.013

43. Ponnusamy M, Liu F, Zhang YH Li RB, Zhai M, Liu F, et al. Long noncoding RNA CPR (Cardiomyocyte Proliferation Regulator) regulates cardiomyocyte proliferation and cardiac repair. Circulation. (2019) 139:266884. doi: 10.1161/CIRCULATIONAHA.118.035832

44. Wang K, Long B, Zhou LY, Liu F, Zhou QY, Liu CY, et al. CARL lncRNA inhibits anoxia-induced mitochondrial fission and apoptosis in cardiomyocytes by impairing miR-539-dependent PHB2 downregulation. Nat Commun. (2014) 5:3596. doi: 10.1038/ncomms4596

45. Li Y, Lin B, Yang L. Comparative transcriptomic analysis of multiple cardiovascular fates from embryonic stem cells predicts novel regulators in human cardiogenesis. Sci Rep. (2015) 5:9758. doi: 10.1038/srep09758
46. Ounzain S, Micheletti R, Arnan C, Plaisance I, Cecchi D, Schroen B, et al. CARMEN, a human super enhancer-associated long noncoding RNA controlling cardiac specification, differentiation and homeostasis. J Mol Cell Cardiol. (2015) 89:98-112. doi: 10.1016/j.yjmcc.2015.09.016

47. Han P, Li W, Lin CH, Yang J, Shang C, Nuernberg ST, et al. A long noncoding RNA protects the heart from pathological hypertrophy. Nature. (2014) 514:102-6. doi: 10.1038/nature13596

48. Wang K, Sun T, Li N, Wang Y, Wang JX, Zhou LY, et al. MDRL IncRNA regulates the processing of miR-484 primary transcript by targeting miR-361. PLoS Genet. (2014) 10:e1004467. doi: 10.1371/journal.pgen.1004467

49. Long QQ, Wang H, Gao W, Fan Y, Li YF, Ma Y, et al. Long noncoding RNA Kcna2 antisense RNA contributes to ventricular arrhythmias via silencing Kcna2 in rats with congestive heart failure. J Am Heart Assoc. (2017) 6:e005965. doi: 10.1161/JAHA.117.005965

50. Zhang YH, Pan X, Zeng T, Chen L, Huang T, Cai YD. Identifying the RNA signatures of coronary artery disease from combined lncRNA and mRNA expression profiles. Genomics. (2020) 112:4945-58. doi: 10.1016/j.ygeno.2020.09.016

51. Holdt LM, Teupser D, Long Noncoding RNA ANRIL. Lnc-ing genetic variation at the chromosome $9 \mathrm{p} 21$ locus to molecular mechanisms of atherosclerosis. Front Cardiovasc Med. (2018) 5:145. doi: 10.3389/fcvm.2018.00145

52. Yari M, Bitarafan S, Broumand MA, Fazeli Z, Rahimi M, Ghaderian SMH, et al. Association between long noncoding RNA ANRIL expression variants and susceptibility to coronary artery disease. Int J Mol Cell Med. (2018) 7:1-7. doi: 10.22088/IJMCM.BUMS.7.1.1

53. Zhang J, Yu L, Xu Y, Liu Y, Li Z, Xue X, et al. Long noncoding RNA upregulated in hypothermia treated cardiomyocytes protects against myocardial infarction through improving mitochondrial function. Int $J$ Cardiol. (2018) 266:213-7. doi: 10.1016/j.ijcard.2017.12.097

54. de Gonzalo-Calvo D, Kenneweg F, Bang C, Toro R, van der Meer RW, Rijzewijk LJ, et al. Circulating long-non coding RNAs as biomarkers of left ventricular diastolic function and remodelling in patients with well-controlled type 2 diabetes. Sci Rep. (2016) 6:37354. doi: 10.1038/srep37354

55. Yang KC, Yamada KA, Patel AY, Topkara VK, George I, Cheema $\mathrm{FH}$, et al. Deep RNA sequencing reveals dynamic regulation of myocardial noncoding RNAs in failing human heart and remodeling with mechanical circulatory support. Circulation. (2014) 129:1009-21. doi: 10.1161/CIRCULATIONAHA.113.003863

56. Zhuo C, Jiang CR, Lin X, Shao M. LncRNA H19 inhibits autophagy by epigenetically silencing of DIRAS3 in diabetic cardiomyopathy. Oncotarget. (2017) 8:1429-37. doi: 10.18632/oncotarget.13637

57. Buxbaum AR, Haimovich G, Singer RH. In the right place at the right time: visualizing and understanding mRNA localization. Nat Rev Mol Cell Biol. (2015) 16:95-109. doi: 10.1038/nrm3918

58. Bridges MC, Daulagala AC, Kourtidis A. LNCcation: lncRNA localization and function. J Cell Biol. (2021) 220:e202009045. doi: 10.1083/jcb.2020 09045

59. Rashid F, Shah A, Shan G. Long Non-coding RNAs in the cytoplasm. Gen Proteom Bioinform. (2016) 14:73-80. doi: 10.1016/j.gpb.2016.03.005

60. Noh JH, Kim KM, McClusky WG, Abdelmohsen K, Gorospe M. Cytoplasmic functions of long noncoding RNAs. Wiley Interdiscip Rev RNA. (2018) 9:e1471. doi: 10.1002/wrna.1471

61. Thomson DW, Dinger ME. Endogenous microRNA sponges: evidence and controversy. Nat Rev Genet. (2016) 17:272-83. doi: 10.1038/nrg.2016.20

62. Song C, Zhang J, Qi H, Feng C, Chen Y, Cao Y, et al. The global view of mRNA-related ceRNA cross-talks across cardiovascular diseases. Sci Rep. (2017) 7:10185. doi: 10.1038/s41598-017-10547-z

63. Bhat SA, Ahmad SM, Mumtaz PT, Malik AA, Dar MA, Urwat U, et al. Long non-coding RNAs: mechanism of action and functional utility. Noncoding RNA Res. (2016) 1:43-50. doi: 10.1016/j.ncrna.2016.11.002

64. White NM, Cabanski CR, Silva-Fisher JM, Dang HX, Govindan R, Maher CA. Transcriptome sequencing reveals altered long intergenic non-coding RNAs in lung cancer. Genome Biol. (2014) 15:429. doi: 10.1186/s13059-014-0429-8

65. Legnini I, Morlando M, Mangiavacchi A, Fatica A, Bozzoni I. A feedforward regulatory loop between HuR and the long noncoding RNA linc-MD1 controls early phases of myogenesis. Molecular cell. (2014) 53:506514. doi: 10.1016/j.molcel.2013.12.012 
66. Guo CJ, $\mathrm{Xu}$ G, Chen LL. Mechanisms of long noncoding RNA nuclear retention. Trends Biochem Sci. (2020) 45:94760. doi: 10.1016/j.tibs.2020.07.001

67. Deevy O, Bracken AP. PRC2 functions in development and congenital disorders. Development. (2019) 146:dev181354. doi: 10.1242/dev.181354

68. Gan L, Yang Y, Li Q, Feng Y, Liu T, Guo W. Epigenetic regulation of cancer progression by EZH2: from biological insights to therapeutic potential. Biomark Res. (2018) 6:10. doi: 10.1186/s40364-018-0122-2

69. Bonnal SC, Lopez-Oreja I, Valcarcel J. Roles and mechanisms of alternative splicing in cancer - implications for care. Nat Rev Clin Oncol. (2020) 17:45774. doi: 10.1038/s41571-020-0350-x

70. Naro C, Sette C. Phosphorylation-mediated regulation of alternative splicing in cancer. Int J Cell Biol. (2013) 2013:151839. doi: 10.1155/2013/151839

71. Wang Y, Liu J, Huang BO, Xu YM Li J, Huang LF, et al. Mechanism of alternative splicing and its regulation. Biomed Rep. (2015) 3:1528. doi: 10.3892/br. 2014.407

72. Song HK, Hong SE, Kim T, Kim DH. Deep RNA sequencing reveals novel cardiac transcriptomic signatures for physiological and pathological hypertrophy. PLoS ONE. (2012) 7:e35552. doi: 10.1371/journal.pone.0035552

73. Peters T, Hermans-Beijnsberger S, Beqqali A, Bitsch N, Nakagawa S, Prasanth $\mathrm{KV}$, et al. Long Non-Coding RNA Malat-1 Is Dispensable during Pressure Overload-Induced Cardiac Remodeling and Failure in Mice. PLoS ONE. (2016) 11:e0150236. doi: 10.1371/journal.pone.0150236

74. Schmitz SU, Grote P, Herrmann BG. Mechanisms of long noncoding RNA function in development and disease. Cell Mol Life Sci. (2016) 73:2491509. doi: 10.1007/s00018-016-2174-5

75. Hang CT, Yang J, Han P, Cheng HL, Shang C, Ashley E, et al. Chromatin regulation by Brg1 underlies heart muscle development and disease. Nature. (2010) 466:62-7. doi: 10.1038/nature09130

76. Saga Y, Kitajima S, Miyagawa-Tomita S. Mesp1 expression is the earliest sign of cardiovascular development. Trends Cardiovasc Med. (2000) 10:34552. doi: 10.1016/S1050-1738(01)00069-X

77. Saga Y, Hata N, Kobayashi S, Magnuson T, Seldin MF, Taketo MM. MesP1: a novel basic helix-loop-helix protein expressed in the nascent mesodermal cells during mouse gastrulation. Development. (1996) 122:276978. doi: 10.1242/dev.122.9.2769

78. Sclafani RA, Holzen TM. Cell cycle regulation of DNA replication. Annu Rev Genet. (2007) 41:237-80. doi: 10.1146/annurev.genet.41.110306.130308

79. C.Gong, Li Z, Ramanujan K, Clay I, Zhang Y, Lemire-Brachat S, et al. A long non-coding RNA, LncMyoD, regulates skeletal muscle differentiation by blocking IMP2-mediated mRNA translation. Dev Cell. (2015) 34:18191. doi: 10.1016/j.devcel.2015.05.009

80. Kurian L, Aguirre A, Sancho-Martinez I, Benner C, Hishida T, Nguyen TB, et al. Identification of novel long noncoding RNAs underlying vertebrate cardiovascular development. Circulation. (2015) 131:1278-90. doi: 10.1161/CIRCULATIONAHA.114.013303

81. Deng H, Ouyang W, Zhang L, Xiao X, Huang Z, Zhu W. LncRNA GASL1 is downregulated in chronic heart failure and regulates cardiomyocyte apoptosis. Cell Mol Biol Lett. (2019) 24:41. doi: 10.1186/s11658-019-0165-x

82. Zhu YJ, Mao D, Gao W, Hu H. Peripheral whole blood lncRNA expression analysis in patients with eosinophilic asthma. Medicine (Baltimore). (2018) 97:e9817. doi: 10.1097/MD.0000000000009817

83. Hermans-Beijnsberger S, van Bilsen M, Schroen B. Long non-coding RNAs in the failing heart and vasculature. Noncoding RNA Res. (2018) 3:118130. doi: 10.1016/j.ncrna.2018.04.002

84. Boeckel JN, Perret MF, Glaser SF, Seeger T, Heumuller AW, Chen W, et al. Identification and regulation of the long non-coding RNA Heat2 in heart failure. J Mol Cell Cardiol. (2019) 126:13-22. doi: 10.1016/j.yjmcc.2018.11.004
85. Dorn GW, LIPCAR. a mitochondrial lnc in the noncoding RNA chain? Circ Res. (2014) 114:1548-50. doi: 10.1161/CIRCRESAHA.114.304 028

86. Derrien T, Johnson R, Bussotti G, Tanzer A, Djebali S, Tilgner H, et al. The GENCODE v7 catalog of human long noncoding RNAs: analysis of their gene structure, evolution, and expression. Genome Res. (2012) 22:1775-89. doi: 10.1101/gr.132159.111

87. Johnsson P, Lipovich L, Grander D, Morris KV. Evolutionary conservation of long non-coding RNAs; sequence, structure, function. Biochim Biophys Acta. (2014) 1840:1063-71. doi: 10.1016/j.bbagen.2013.10.035

88. Li DY, Busch A, Jin H, Chernogubova E, Pelisek J, Karlsson J, et al. H19 induces abdominal aortic aneurysm development and progression. Circulation. (2018) 138:1551-68. doi: 10.1161/CIRCULATIONAHA.117.0 32184

89. Huang S, Zhang L, Song J, Wang Z, Huang X, Guo Z, et al. Long noncoding RNA MALAT1 mediates cardiac fibrosis in experimental postinfarct myocardium mice model. J Cell Physiol. (2019) 234:29973006. doi: $10.1002 /$ jcp. 27117

90. Micheletti R, Plaisance I, Abraham BJ, Sarre A, Ting CC, Alexanian M, et al. The long noncoding RNA Wisper controls cardiac fibrosis and remodeling. Sci Transl Med. (2017) 9:eaai9118. doi: 10.1126/scitranslmed.aai9 118

91. Tao H, Zhang JG, Qin RH Dai C, Shi P, Yang JJ, et al. LncRNA GAS5 controls cardiac fibroblast activation and fibrosis by targeting miR-21 via PTEN/MMP-2 signaling pathway. Toxicology. (2017) 386:118. doi: 10.1016/j.tox.2017.05.007

92. Piccoli MT, Gupta SK, Viereck J, Foinquinos A, Samolovac S, Kramer FL, et al. Inhibition of the cardiac fibroblast-enriched lncRNA Meg3 prevents cardiac fibrosis and diastolic dysfunction. Circ Res. (2017) 121:575-83. doi: 10.1161/CIRCRESAHA.117.3 10624

93. Huang CK, Kafert-Kasting S, Thum T. Preclinical and clinical development of noncoding RNA therapeutics for cardiovascular disease. Circ Res. (2020) 126:663-78. doi: 10.1161/CIRCRESAHA.119.3 15856

94. Taubel J, Hauke W, Rump S, Viereck J, Batkai S, Poetzsch J, et al. Novel antisense therapy targeting microRNA-132 in patients with heart failure: results of a first-in-human Phase $1 \mathrm{~b}$ randomized, double-blind, placebo-controlled study. Eur Heart J. (2021) 42:178-88. doi: 10.1093/eurheartj/ehaa898

Conflict of Interest: The authors declare that the research was conducted in the absence of any commercial or financial relationships that could be construed as a potential conflict of interest.

Publisher's Note: All claims expressed in this article are solely those of the authors and do not necessarily represent those of their affiliated organizations, or those of the publisher, the editors and the reviewers. Any product that may be evaluated in this article, or claim that may be made by its manufacturer, is not guaranteed or endorsed by the publisher.

Copyright (c) 2021 Han and Yang. This is an open-access article distributed under the terms of the Creative Commons Attribution License (CC BY). The use, distribution or reproduction in other forums is permitted, provided the original author(s) and the copyright owner(s) are credited and that the original publication in this journal is cited, in accordance with accepted academic practice. No use, distribution or reproduction is permitted which does not comply with these terms. 